\title{
OBESITY
}

\section{Does bariatric surgery improve offspring health?}

Metabolic benefits of bariatric surgery in women might not extend to metabolic benefits in their offspring, new data reveal.

Grayson et al. performed vertical sleeve gastrectomies (VSG) on female rats rendered obese with a high-fat diet to answer two questions. "First, are the improvements after bariatric surgery similar in female rats to those seen previously in male rats?", explains senior author Randy Seeley. "Second, if female rats improve their weight and metabolic health, will that result in improvements in the risk of metabolic problems in their offspring?"

Female rats fed either a high-fat diet or normal chow after surgery lost weight and body fat and had improved glucose tolerance. Offspring of rats that had undergone VSG (irrespective of the type of maternal diet after surgery) were born small-for-gestation age in comparison with offspring from lean or obese mice that had undergone sham surgery.
Importantly, offspring of rats that had undergone VSG challenged with a high-fat diet in adulthood became more glucose intolerant and had greater adiposity than offspring of lean or obese rats that had undergone sham surgery.

The findings might have important clinical implications. "Maternal weight loss and metabolic improvements typically reduce risk in offspring, but that was not the case when those improvements happened as a result of VSG," says Seeley. "Future work will need to address whether this effect is specific to this type of weight loss surgery and whether it applies in humans."

\section{Carol Wilson}

Original article Grayson, B. E. et al. Improved rodent maternal metabolism but reduced intrauterine growth after vertical sleeve gastrectomy. Sci. Transl. Med. 5, 199ra112 (2013) 